\title{
Effect of Environment on the Underlying Stellar Populations of Dwarf Elliptical Galaxies in the Coma Cluster
}

\author{
A. Matković*, R. Guzmán*, P. Sánchez-Blázquez ${ }^{\dagger}$, N. Cardiel \\ ** and J. Gorgas** \\ *University of Florida, 211 Bryant Space Science Center P.O. Box 112055, Gainesville, FL, \\ 32611-2055, USA \\ †Centre for Astrophysics, University of Central Lancashire, PR1 2HE, Preston, UK \\ ${ }^{* *}$ Departamento de Astrofisica, Facultad de Fisicas, Universidad Complutense de Madrid, Ciudad \\ Universitaria, 28040 Madrid, Spain
}

\begin{abstract}
We derive ages, metallicities and $[\alpha / \mathrm{Fe}]$ ratios for dwarf early-type galaxies $(\mathrm{dE} / \mathrm{dS} 0)$ in the center and a region believed to be infalling into the Coma cluster. We find that a set of metallic indices cannot be described by linear relations with velocity dispersion $(\sigma)$, while another group of indices exhibits linear index- $\sigma$ relations. $\mathrm{dE} / \mathrm{dS} 0$ galaxies in the core of the cluster are on average younger, and have lower metallicities and lower $\alpha$-ratios (solar to sub-solar) than the more luminous elliptical galaxies. On the other hand, $\mathrm{dEs} / \mathrm{dS} 0 \mathrm{~s}$ in the infall region of Coma form a less homogeneous population. We find an unusually high fraction of $\mathrm{dE} / \mathrm{dS} 0 \mathrm{~s}$ with high $[\alpha / \mathrm{Fe}]$ ratios, suggesting short time scales for the star formation histories for these galaxies. Furthermore, the $\mathrm{dE} / \mathrm{dSO}$ s with high $\alpha$-ratios have a range of ages and metallicities implying multiple formation scenarios where some galaxies have experienced their short star formation bursts at more recent epochs.
\end{abstract}

Keywords: dwarf galaxies, early-type galaxies, stellar population, Coma cluster, environment, line strength

PACS: $98.52 . \mathrm{Wz}$, 98.52.Eh, 98.62.-g, 98.65. CW

\section{INTRODUCTION}

Dwarf elliptical galaxies are the most numerous galaxies in clusters. Since their potential wells are shallow, dEs are susceptible to a wide range of processes. This makes them ideal testbeds for different galaxy formation and evolution scenarios. Currently favored mechanisms for $\mathrm{dE}$ galaxies in clusters include the galaxy harassment scenario, where spiral galaxies are transformed into $\mathrm{dE} / \mathrm{dS} 0 \mathrm{~s}$ due to fly-by interactions of galaxies in the cluster (Moore, Katz \& Lake 1996), and scenarios where dEs are the evolved counterparts of dwarf irregular galaxies, dIs, (e.g. Dekel \& Silk 1986). In both scenarios, the progenitors of $\mathrm{dE} / \mathrm{dS} 0$ galaxies undergo gas-loss and morphological transformation, whether it is through interactions with other galaxies in the cluster, or via ram pressure stripping as they enter the cluster environment (Lin \& Faber 1983). Signatures of these events can be seen in the stellar populations of dEs since their ages, metallicities and chemical abundances depend on the amount of time passed between the star formation event and the point in which they entered the cluster, or whether their star formation occurred before or after accretion into the cluster. 

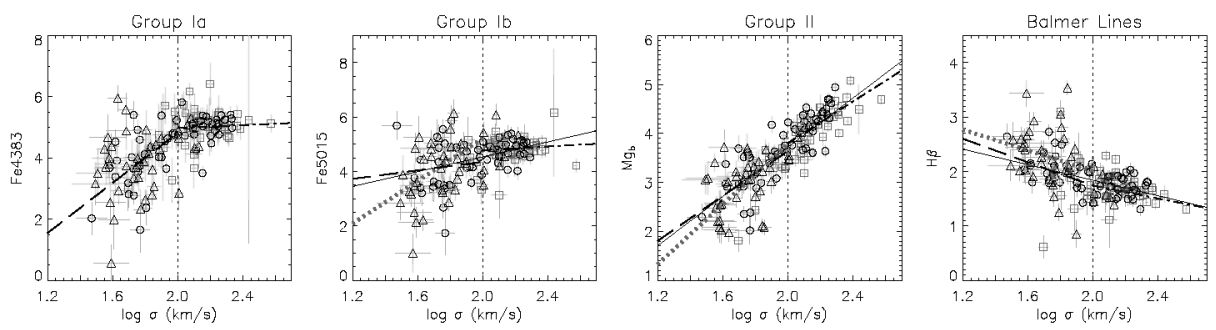

FIGURE 1. Representative Index-log $\sigma$ plots. Open circles and triangles are our central and outer region galaxies, respectively, while the open squares are Coma cluster galaxies from the NFPS survey. Each plot here represents a group of $I-\sigma$ relations described in the text and presented in full format in Matković (2008). The solid lines represent linear fits for all the galaxies in the individual $I-\sigma$ figures, while the dashed and dash-dotted line mark linear fits to low- and high- $\sigma$ galaxies respectively.

The different formation models predict the properties of the underlying stellar populations of $\mathrm{dE} / \mathrm{dS} 0$ galaxies depending on their location in the cluster. For example, the galaxy harassment scenario predicts that $\mathrm{dE} / \mathrm{dSOS}$ found in the center of the cluster would have higher metallicities than galaxies in the lower-density environments within the cluster. On the other hand, the model where dwarfs form from accreted field galaxies predicts older ages and lower metallicities in the center of the cluster.

In this work, we investigate the underlying stellar populations of $\sim 70 \mathrm{dE} / \mathrm{dS} 0$ galaxies in two regions within the Coma cluster. Our sample includes early-type galaxies in the center of the cluster, a high density region, and galaxies in a region of lower density, centered around NGC 4839 which is believed to be falling into the cluster. Our aim here is to investigate which formation scenario(s) best reproduce properties of dEs in clusters, whether cluster $\mathrm{dE} / \mathrm{dS} 0$ s have a homogeneous formation and what role does the environment play in shaping the properties of the $\mathrm{dE} / \mathrm{dS} 0$ population.

\section{INDEX- $\sigma$ RELATIONS}

We find that $I-\sigma$ relations for metallic indices differ in shape for low- and high- $\sigma$ galaxies ( $\sigma<100$ and $\sigma \geq 100 \mathrm{~km} \mathrm{~s}^{-1}$, respectively) depending on the index. We divide these relations into group I and group II where the first set of indices exhibits evidence for a break in the slope around $\sigma 100 \mathrm{~km} \mathrm{~s}^{-1}$, while the other group of indices shows robust linear relations with $\log \sigma$ (Matković et al. 2008). Group I contains a set of indices where the evidence for a break in the slope is stronger, group Ia, than for another set of indices, group $\mathrm{Ib}$. We only show a subset of $I-\sigma$ relations in Figure 1. For a figure including all the indices in this study, please see Matković et al. (2008) and Matković (2008). We find no connection between either age, or metallicity, or $[\alpha / \mathrm{Fe}]$ with the shape of the $I-\sigma$ relations. However, indices in group II are independent of microturbulent velocity of stellar atmospheres (Trippico \& Bell 1995) which may turn out to be a key factor in determining the shape of $I-\sigma$ relations.

Galaxies in the outer region of the Coma cluster exhibit the same $I-\sigma$ relations as the $\mathrm{dE} / \mathrm{dS} 0 \mathrm{~s}$ in the center. However, for most indices the scatter of outer galaxies is larger 

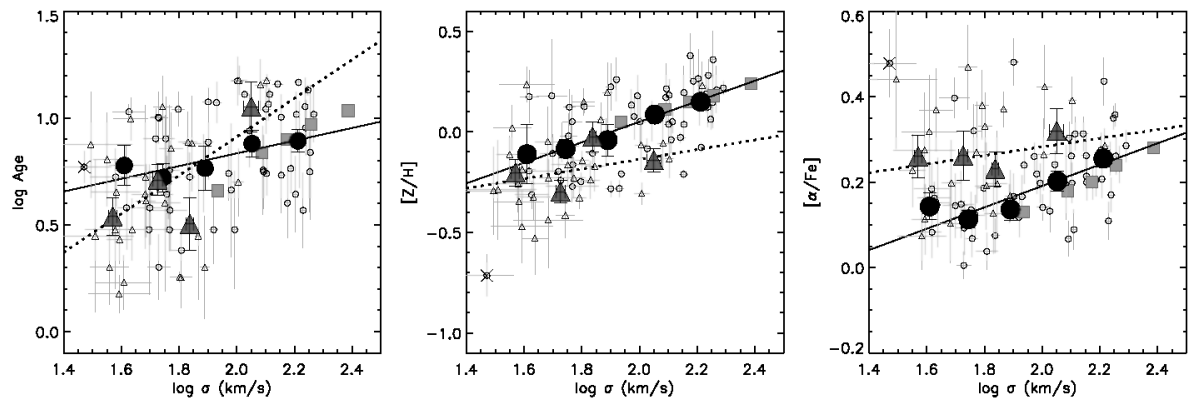

FIGURE 2. Environmental effects on $\sigma$ vs. age, metallicity and [ $\alpha / \mathrm{Fe}]$. Small open circles and triangles represent measurements for individual galaxies for the central and the outer region of the cluster, respectively. In the same order we show large filled circles and triangles which correspond to the average value of the SPM parameter at a given $\sigma$. The solid line corresponds to the linear fit for the central binned galaxies, and the dotted line to the outer data. Note that, since there is only one galaxy in the highest $\sigma$ bin for the outer sample, we did not assign it a bin. NFPS data are marked by grey filled squares.

than it is for the central galaxies. Similarly, the Balmer lines show a larger scatter for $\mathrm{dE} / \mathrm{dSO}$ galaxies in the outskirts than in the center. Further, there are more galaxies with low $\mathrm{H} \beta$ in the outer sample which may imply younger ages for $\mathrm{dE} / \mathrm{dS} 0 \mathrm{~s}$ in the region believed to be infalling into the cluster.

\section{PROPERTIES OF STELLAR POPULATIONS}

We used the models of Thomas, Maraston and Korn (2004) to derive the ages, metallicities and $[\alpha / \mathrm{Fe}]$. We find that the high- $\sigma$ galaxies have more uniform metallicities than the low- $\sigma$ galaxies which show a wide range in all stellar population model (SPM) parameters (age, metallicity and $\alpha$-ratios).

In Figure 2, we examine the relation between the SPM parameters and $\sigma$. To reduce correlated errors derived by models, we used average values of each index which was binned by $\sigma$. The linear relations were derived based on the binned data which is represented by solid circles for the center galaxies and triangles for galaxies in the infall region of the cluster. Our derived relations are consistent with the NOAO Fundamental Plane Survey, NFPS, (Smith et al. 2004) also presented in the figure.

Galaxies in the center of Coma show a weak trend where the low- $\sigma$ galaxies are, on average, younger than the high- $\sigma$ early-types. Similarly, metallicity increases with $\sigma$ for the central galaxies, where the more massive elliptical galaxies are more metal rich than the $\mathrm{dE} / \mathrm{dSOs}$. We find a hint toward younger ages and lower metallicities for $\mathrm{dE} / \mathrm{dSO}$ in the infalling region of Coma when compared to the galaxies in the center of the cluster.

We note a larger scatter in all parameters for galaxies in the infalling region. This implies that galaxies in the center of the Coma cluster may have more homogeneous stellar populations when compared to the galaxies in the infall region. The largest environmental effect that we find between SPM parameters and $\sigma$ is in the $\alpha$-ratios. The infall $\mathrm{dE} / \mathrm{dSO}$ s have, on average, higher $[\alpha / \mathrm{Fe}]$ ! This is a surprising result, since 
$\mathrm{dE} / \mathrm{dS} 0$ galaxies are considered to have sub-solar to solar $\alpha$-ratios. Further examination of $\mathrm{dE} / \mathrm{dS} 0 \mathrm{~s}$ with high $\alpha$-ratios revealed three groups of these objects. One set of galaxies with high $\alpha$-ratios exhibits old ages and low metallicities, another set young ages and high metallicities, and the third group has young ages and low metallicities. Most of these characteristics of $\mathrm{dE} / \mathrm{dS} 0 \mathrm{~s}$ with high $[\alpha / \mathrm{Fe}]$ can be explained by different scenarios. For example, dIs falling into the cluster right after a short starburst would lose their gas through ram pressure stripping. In this case, we would observe high $\alpha$-element abundances and low metallicities, since these galaxies would not have enough time to incorporate Fe from Supernova Type Ia into the new generation of stars. In conclusion, galaxies in the infalling region of the cluster have heterogeneous star formation histories, while $\mathrm{dE} / \mathrm{dSO}$ galaxies in the center of the cluster show more homogeneous stellar populations.

\section{SUMMARY}

We investigated the stellar populations of dwarf early-type galaxies in two regions of different density within the Coma cluster. We find that the index- $\sigma$ relations for most metallic indices show a change of slope in the dwarf regime. Dwarf early-type galaxies also show general trends of younger ages and higher metallicities than more massive ellipticals.

Galaxies in the center of the cluster are, on average, older and slightly more metal rich than the ones found in the region believed to be falling into the cluster. Our central $\mathrm{dE} / \mathrm{dS} 0 \mathrm{~s}$ are in agreement with previous studies showing solar to sub-solar $\alpha$ ratios. However, we find that a number of these galaxies in the infall region have $[\alpha / \mathrm{Fe}]$ similar to gEs. This suggests short time scales in their star formation histories, where some $\mathrm{dE} / \mathrm{dSO}$ experience a short burst long time ago, and others very recently. In summary, dwarf early-type galaxies in the center of the cluster have a more homogeneous star formation history, while the outer galaxies are a heterogeneous population.

\section{ACKNOWLEDGMENTS}

I thank the SOC for financial support to attend the conference.

\section{REFERENCES}

1. Dekel, A., \& Silk, J., 1986, ApJ, 303, 39

2. Lin, D. N. C., \& Faber, S. M., 1983, ApJ, 266, L21

3. Matković, A., Guzmán, R., Sánchez-Blázquez, P., Gorgas, J., Cardiel, N., and Gruel, N., accepted to ApJ, arXiv:0810.3237

4. Matković, A. 2008, Ph.D. Dissertation, Internal Kinematics and Stellar Populations of Dwarf EarlyType Galaxies in the Coma Cluster

5. Moore, B., Katz, N., and Lake, G. 1996, ApJ, 457, 455

6. Smith, R.J., Hudson, M.J., Nelan, J.E., Moore, S.A., Quinney, S.J., and 6 co-authors 2004, AJ, 128, $1558 \mathrm{~S}$

7. Thomas, D., Maraston, C., \& Korn, A. 2004, MNRAS, 351, L19

8. Trippico, M. J., \& Bell, R. A. 1995, AJ, 110,3035 
Copyright of AIP Conference Proceedings is the property of American Institute of Physics and its content may not be copied or emailed to multiple sites or posted to a listserv without the copyright holder's express written permission. However, users may print, download, or email articles for individual use. 